\title{
Método simplificado para estimativa das dimensões do bulbo molhado na irrigação por gotejamento superficial
}

O conhecimento das dimensões do bulbo molhado do solo na irrigação por gotejamento superficial é um aspecto importante que vem chamando a atenção de pesquisadores pelo fato de definir tanto a lâmina e a frequência de irrigação, como também o número de gotejadores e o dimensionamento do sistema. Para isso, diversos modelos foram utilizados em diferentes trabalhos, destacando o modelo potencial e o de superfície de resposta para estimar as dimensões do bulbo em condições experimentais em função da vazão do gotejador e/ou tempo de aplicação de água. Nesse sentido, objetivou-se com este trabalho propor, ajustar e avaliar modelos matemáticos para estimar diâmetro máximo (Dmax), profundidade máxima (Zmax) e diâmetro superficial (Ds) do bulbo molhado usando o volume de 1,0 L de água. Para isso foram instalados seis experimentos em diferentes solos e avaliadas as dimensões do bulbo em função do volume de água aplicado. Foram propostos três modelos, sendo os valores de Dmax, Zmax e Ds para a aplicação de 1,0 L de água. Concluiu-se que os modelos estimaram satisfatoriamente as dimensões avaliadas, com coeficiente de determinação (R2) entre os valores observados e estimados variando de 0,9615 a 0,9896, 0,9212 a 0,9835 e 0,8904 a 0,9704 para Dmax, Zmax e Ds, respectivamente.

\section{Simplified method to estimate the wetted bulb dimensions in surface drip irrigation}

\begin{abstract}
The knowledge of the dimensions of the wetted soil surface in drip irrigation is an important aspect that causes the attention of researchers because it defines filtration and irrigation frequency, as well as the number of emitters and system design. For this many models have been used in different studies, highlighting the potential model and response surface to estimate the dimensions of the bulb on the experimental conditions in relation to the emitter discharged and time of water application. Accordingly, the aim of this work was propose, adjust and evaluate mathematical models to estimate the maximum diameter (Dmax), maximum depth (Zmax) and surface diameter (Ds) using the wet bulb volume of $1.0 \mathrm{~L}$ of water. For six experiments that were installed in different soils and evaluated the size of the bulb due to the volume of water applied. Three models were proposed, and the values of Dmax, Zmax and Ds for applying 1.0 L of water. It was concluded that the models satisfactorily estimated dimensions assessed, with a coefficient of determination (R2) between observed and estimated values ranging from 0.9615 to $0.9896,0.9212$ to 0.9835 and from 0.8904 to 0.9704 to Dmax, Zmax and Ds, respectively.
\end{abstract}

Keywords: Soil and Water Modeling; Soil Water Infiltration; Localized Irrigation.

Topic: Engenharia Agrícola

Reviewed anonymously in the process of blind peer.

Celsemy Eleutério Maia

Universidade Federal Rural do Semi-Árido, Brasil

http://lattes.cnpq.br/1217682039861125

http://orcid.org/0000-0002-2253-3022

celsemy@ufersa.edu.br

José Mariano da Silva Neto (it)

Universidade Federal Rural do Semi-Árido, Brasil

http://lattes.cnpq.br/1035801819440482

http://orcid.org/0000-0002-2427-1855

neto-silva@hotmail.com

Ana Quézia Carvalho Braga (iD

Universidade Federal Rural do Semi-Árido, Brasil

http://lattes.cnpq.br/9196532878983547

http://orcid.org/0000-0002-9859-1720

queziaana@outlook.com
Received: 07/02/2020

Approved: 04/03/2020
Referencing this:

MAIA, C. E.; SILVA NETO, J. M.; BRAGA, A. Q. C.. Método simplificado para estimativa das dimensões do bulbo molhado na irrigação por gotejamento superficial. Revista Ibero Americana de Ciências Ambientais, v.11, n.2, p.53-61, 2020. DOI:

http://doi.org/10.6008/CBPC2179-6858.2020.002.0006 


\section{INTRODUÇÃO}

A irrigação por gotejamento tem sido amplamente difundida em todo mundo, principalmente, em regiões áridas e semiáridas, fato atribuído a seus bons benefícios com relação à economia de água (YANG et al., 2020). De acordo com Chandramohan et al. (2019) é de fundamental importância o estudo de uma tecnologia eficiente de irrigação por gotejamento associado a conservação de energia para essas regiões em que há pouca disponibilidade de água, assim, a irrigação por gotejamento tem desempenhado um papel importante com relação a recursos hídricos e a otimização da eficiência do uso de água nessas regiões (KANG et al., 2017).

De acordo com Ma et al. (2020) entre os diferentes tipos de irrigação por gotejamento, a irrigação localizada por gotejamento ou superficial destaca-se e é amplamente adotada devido ao seu fácil processo de instalação e manutenção. Nesse tipo de irrigação há um melhor gerenciamento de água, potencial economia de energia, melhor controle de ervas daninhas, melhoria de rendimento e qualidade, custos mais baixos e problemas reduzidos de insetos (SCHWANKL et al., 2007).

Um dos aspectos mais importantes nesse tipo de irrigação é o dimensionamento do bulbo molhado do solo. A partir do dimensionamento é possível definir a lâmina e frequência de irrigação, o número de gotejadores e o dimensionamento hidráulico (COOK et al., 2006), como também no manejo da irrigação (HAO et al., 2007).

Um dos principais modelos utilizados para estimar as dimensões do bulbo molhado na irrigação por gotejamento superficial em função da vazão do emissor $(q)$ ou do tempo de aplicação de água $(t)$ é o modelo potencial $\left(y=\alpha \cdot x^{\beta}\right)$, comumente utilizados e com bons resultados, como se pode observar nos trabalhos de Thorburn et al. (2003), Li et al. (2004), Thabet et al. (2008), Maia et al. (2010) e Bezerra et al. (2015)

Uma característica do modelo potencial é que a taxa de aumento das dimensões do bulbo molhado é maior para as vazões mais baixas e menores tempos de irrigação, diminuindo com o aumento de q e t. Maia et al. (2010) avaliando as dimensões do diâmetro superficial $\left(D_{s}\right)$, diâmetro máximo $\left(D_{\max }\right)$, profundidade máxima $\left(Z_{\max }\right)$ e profundidade onde ocorre $D_{\max }\left(Z_{D \max }\right)$, em seis solos avaliados em condição de campo, verificaram que para quatro valores de q e quatro de $t$, foram necessárias 196 equações potenciais para estimar todas as dimensões desejadas para os seis solos.

Nesse tipo de estudo é necessário fixar os valores de $t$ e estimar as dimensões em função de $q$ ou vice-versa, ou seja, $y=\alpha \cdot t^{\beta}$ ou $y=\alpha \cdot q^{\beta}$, levando a um maior número de equações para estimar as dimensões nas condições do estudo. Na sequência, Maia et al. (2010) propuseram a utilização do modelo de superfície de resposta $\left(y=a \cdot t^{b} \cdot q^{c}\right)$, em que, para as condições do estudo de Maia et al. (2010), o número de equações para as mesmas condições, diminuiu das 196 para apenas 24 equações.

Nesse sentido, a utilização do modelo potencial $y=\alpha \cdot x^{\beta}$, apesar de apresentar apenas dois parâmetros ( $\alpha$ e 6 ) teria que fixar $q$ e estimar $t$ ou o contrário, limitando seu uso para os valores de $q$ ou $t$ fixado. Utilizando o modelo de superfície de resposta, apesar do modelo apresentar três parâmetros $(a, b$ e c), estima-se as dimensões do bulbo para quaisquer valores de $q$ e $t$ dentro do espaço experimental. 
Posteriormente, Maia (2011), visando diminuir o número de parâmetros dos modelos avaliados para estimar as dimensões do bulbo molhado, levando em consideração que o produto de $q$ e $t$ é o volume de água aplicado $\left(V_{a p}\right)$, avaliou o modelo potencial $y=a \cdot V_{a p}{ }^{b}$ e observou que, com apenas dois parâmetros ( $a$ e b), um parâmetro a menos que o modelo de superfície de resposta, os resultados foram semelhantes aos de Maia et al. (2010).

Assim, o objetivo do presente trabalho foi avaliar o modelo potencial com a estimativa de apenas um parâmetro para avaliar as dimensões do bulbo molhado em função do volume de água aplicado na irrigação localizada superficial, como também diferentes métodos para estimar este parâmetro em seis solos do Agropólo Assu-Mossoró.

\section{METODOLOGIA}

Para avaliar o modelo simplificado foram utilizados dados de Maia et al. (2010), onde a região do estudo é caracterizada, segundo a classificação de Köppen, como BSwh', isto é, seco, muito quente e com estação chuvosa no verão atrasando-se para o outono, apresentando temperatura média anual de $27,4^{\circ} \mathrm{C}$, precipitação pluviométrica anual bastante irregular, com média de $673,9 \mathrm{~mm}$, e umidade relativa de $68,9 \%$ (CARMO FILHO et al., 1991). Foram selecionados seis solos para o referido estudo, sendo classificados segundo Santos et. al. (2013) como Luvissolo Crômico (S1), Argissolo Vermelho-Amarelo (S2), Cambissolo Háplico (S3), Neossolo Quartizarênico (S4), Latossolo Vermelho (S5) e Neossolo Flúvico (S6).

Antes da montagem do sistema de irrigação, os solos foram preparados de forma a simular as reais condições de plantio, com aração e passagem da grade de disco de forma cruzada. Para determinar com maior exatidão o tamanho do bulbo molhado (diâmetro da área molhada e profundidade) foi desenvolvido um sistema de irrigação portátil, onde foram dispostos oito emissores por linha, distribuídos aleatoriamente na parcela, ao longo de uma tubulação de polietileno de $16 \mathrm{~mm}$ de diâmetro, abastecida por dois depósitos de água.

Para regular a vazão e a pressão, foram utilizados reservatórios reguladores, conectados aos primeiros, que mantinham um nível constante através de um sistema de boia, sendo sua haste alterada de forma que sua carga hidráulica se mantivesse estável. As diferentes vazões foram obtidas pelos diferentes comprimentos dos microtubos inseridos na tubulação de polietileno. Estes, juntamente com a carga hidráulica constante, geraram diferentes vazões nas saídas dos mesmos, que eram fechados quando concluído cada tempo de irrigação. O espaçamento entre os emissores foi para que não houvesse sobreposição, ou seja, interferência dos emissores laterais.

Os experimentos foram instalados em delineamento em blocos inteiramente casualizados, no esquema de parcela subdividida, com três repetições, cujos tratamentos foram compostos pelos fatores tempo de aplicação de água e vazão do emissor, sendo o tempo, a parcela e a vazão, a subparcela. As repetições constaram de duas linhas de irrigação com quatro diferentes tempos (1, 2, 4 e 7 h) e, dentro de cada tempo, as vazões de $1,2,4$ e $8 \mathrm{~L} \mathrm{~h}^{-1}$ obtidas por microtubos de tamanhos diferenciados que 
proporcionam as vazões desejadas sob as mesmas condições de carga hidráulica. Para cada vazão, imediatamente após os tempos pré-determinados de 1, 2, 4 e 7 h, foram abertas trincheiras no centro do bulbo molhado, abaixo do emissor, onde se realizou as medições das dimensões do bulbo com fita métrica com precisão de $1,0 \mathrm{~mm}$.

Para avaliar o efeito da vazão do emissor $(q)$ e do tempo de aplicação de água $(t)$ na formação do bulbo molhado avaliaram-se as seguintes características: diâmetro superficial $\left(D_{s}\right)$, diâmetro máximo $\left(D_{\max }\right)$, profundidade máxima $\left(Z_{\max }\right)$ e profundidade onde se verificou $D_{\max }\left(Z_{D \max }\right)$. Com os dados observados ajustouse o modelo potencial $y=a \cdot V_{a p}{ }^{b}$, sendo $y$ as dimensões do bulbo $(\mathrm{cm}), V_{a p}$ (produto de $q\left(\mathrm{~L} \mathrm{~h}^{-1}\right)$ e $\left.t(\mathrm{~h})\right)$ o volume de água aplicado (L) e $a$ e $b$ os parâmetros do modelo, sendo $b$ fixado de acordo com a dimensão avaliada, como deduzido a seguir. Sendo as dimensões do bulbo molhado $\left(D_{\max }, Z_{\max }, D_{s}\right.$ e $\left.Z_{D \max }\right)$ medidas em comprimento L, a condutividade hidráulica saturada $\left(K_{s}\right)$ em $\mathrm{L} \mathrm{T}^{-1}$ e a vazão do emissor $(q)$ em $\mathrm{L}^{3} \mathrm{~T}^{-1} \mathrm{e}, \mathrm{partindo}$ da equação 1:

$$
D_{\max }=K \cdot Z_{\max }^{1 / 3} \cdot\left(\frac{q}{K_{s}}\right)^{1 / 3}
$$

Com base no trabalho de Schwartzman et al. (1986), rearranjando se tem a equação 2 , sendo $K$ adimensional.

$$
D_{\max }=K \cdot q^{1 / 3} \cdot\left(\frac{Z_{\max }}{K_{s}}\right)^{1 / 3}
$$

Seguindo a dedução:

$$
D_{\max }=K \cdot\left(\frac{L^{3}}{T}\right)^{1 / 3} \cdot\left(\frac{L}{L / T}\right)^{1 / 3} \rightarrow D_{\max }=K \cdot\left(\frac{L^{3}}{T}\right)^{1 / 3} \cdot T^{1 / 3} \rightarrow L=K \cdot\left(\frac{L^{3}}{T} \cdot T\right)^{1 / 3}
$$

Como o produto de $q$ e $t$ é o volume de água aplicado $\left(V_{a p}\right.$ em $\left.L^{3}\right)$ e para $K_{1}$ adimensional, tem-se a equação 3.

$$
D_{\max }=K_{1} \cdot V_{a p}^{1 / 3}
$$

Após a verificação que $Z_{\max }$ e $D_{s}$ em função do $V_{a p}$ se ajustam bem ao modelo potencial, obtive-se os expoentes das equações 4 e 5 .

$$
\begin{aligned}
& Z_{\max }=K_{2} \cdot V_{a p}^{2 / 5} \\
& D_{s}=K_{3} \cdot V_{a p}^{1 / 4}
\end{aligned}
$$

Com, $K_{2}$ e $K_{3}$ em L L ${ }^{-1 / 5} \mathrm{e} \mathrm{L}^{1 / 4}$, respectivamente.

Para cada dimensão avaliada, foram estimados os valores do parâmetro $K$ da equação potencial $y=K \cdot V_{a p}^{b}$ de quatro maneiras $\left(K_{1}^{\prime}, K_{2}^{\prime}, K_{3}^{\prime}\right.$ e $\left.K^{\prime}{ }_{4}\right): K^{\prime}{ }_{1}$ valor estimado por regressão para o modelo $y=K \cdot V_{a p}{ }^{b} ; K^{\prime}{ }_{2}$ obtido pela média de três repetições da dimensão obtidas no experimento de campo para 
$V_{a p}$ de 1,0 L, $K^{\prime}{ }_{3}$ e $K_{4}^{\prime}$ são coeficientes angulares das retas $y=a+b x$ e $y=b x$, respectivamente, com $y$ sendo a dimensão do bulbo e $x$ o valor de $V_{a p}{ }^{1 / 3}, V_{a p}{ }^{2 / 5}$ e $V_{a p}{ }^{1 / 4}$ para $D_{\max }, Z_{\max }$ e $D_{s}$, respectivamente.

\section{RESULTADOS E DISCUSSÃO}

Os valores de $K^{\prime}{ }_{1}, K^{\prime}{ }_{2}, K^{\prime}{ }_{3}$ e $K^{\prime}{ }_{4}$ para os solos estudados, com suas respectivas média, desvio padrão e $\mathrm{CV}$, são mostrados na Tabela 1, em que se observa os menores valores médios de CV foram verificados para $D_{\max }$ e que, independente da dimensão avaliada, o Neossolo Flúvico (S6) foi quem apresentou os maiores valores de CV para os valores de $K^{\prime}{ }_{1}, K_{2}^{\prime}{ }_{2} K^{\prime}{ }_{3}$ e $K^{\prime}{ }_{4}$. Nesse sentido, com exceção do solo $\mathrm{S} 6$, os valores de $\mathrm{CV}$ variaram entre $1,75 \%$ e $4,70 \%$ para o $D_{\max }, 2,30 \%$ a $7,99 \%$ para $Z_{\max }$ e entre $3,23 \%$ a $13,39 \%$ para o $D_{s}$. Comparando os solos, as menores variações entre os valores de K' foi observado para $D_{\max }$ e $D_{s}$ no S2 e para $Z_{\max }$ no $S 4$.

Tabela 1: Valores da constante $K$ do modelo $y=K \cdot V_{a p}{ }^{b}$ obtidos por diferentes critérios para $D_{\max }, Z_{\max }$ e $D_{s}$, média, desvio padrão e coeficiente de variação (CV) dos valores de $K^{\prime}$ para os solos avaliados

\begin{tabular}{|c|c|c|c|c|c|c|c|}
\hline \multirow[t]{2}{*}{ Solo } & \multicolumn{7}{|c|}{$D_{\max }=K_{n}^{\prime} V_{a p}{ }^{1 / 3}$} \\
\hline & $K_{1}^{\prime}$ & $K_{2}^{\prime}$ & $K_{3}^{\prime}$ & $K_{4}^{\prime}$ & Média & Desvio & CV (\%) \\
\hline S1 & 25,42 & 25,24 & 22,98 & 23,97 & 24,40 & 1,15 & 4,70 \\
\hline S2 & 25,02 & 25,48 & 24,49 & 24,67 & 24,92 & 0,44 & 1,75 \\
\hline S3 & 24,11 & 23,31 & 25,17 & 24,85 & 24,36 & 0,83 & 3,40 \\
\hline S4 & 25,50 & 26,06 & 24,39 & 24,66 & 25,15 & 0,77 & 3,05 \\
\hline S5 & 21,90 & 21,44 & 23,42 & 23,18 & 22,49 & 0,96 & 4,29 \\
\hline S6 & 20,34 & 22,48 & 27,97 & 24,31 & 23,78 & 3,23 & 13,60 \\
\hline Média & 23,72 & 24,00 & 24,74 & 24,27 & & & \\
\hline Desvio & 2,13 & 1,86 & 1,77 & 0,62 & & & \\
\hline \multirow[t]{2}{*}{ CV (\%) } & 8,97 & 7,75 & 7,15 & 2,56 & & & \\
\hline & \multicolumn{7}{|c|}{$Z_{\max }=K_{n}^{\prime} V_{a p^{2 / 5}}^{2 / 5}$} \\
\hline S1 & 15,06 & 15,00 & 13,45 & 14,02 & 14,38 & 0,78 & 5,45 \\
\hline S2 & 16,99 & 20,00 & 17,46 & 17,07 & 17,88 & 1,43 & 7,99 \\
\hline S3 & 13,80 & 14,50 & 15,17 & 14,78 & 14,56 & 0,58 & 3,97 \\
\hline S4 & 14,64 & 15,00 & 15,36 & 15,37 & 15,09 & 0,35 & 2,30 \\
\hline S5 & 15,52 & 14,50 & 17,19 & 16,06 & 15,82 & 1,12 & 7,08 \\
\hline S6 & 10,33 & 9,00 & 4,53 & 7,33 & 7,80 & 2,50 & 32,07 \\
\hline Média & 14,39 & 14,67 & 13,86 & 14,11 & & & \\
\hline Desvio & 2,25 & 3,49 & 4,80 & 3,48 & & & \\
\hline \multirow[t]{2}{*}{ CV (\%) } & 15,66 & 23,78 & 34,64 & 24,68 & & & \\
\hline & \multicolumn{7}{|c|}{$D_{s}=K_{n}^{\prime} V_{a p}^{1 / 4}$} \\
\hline S1 & 25,29 & 24,96 & 23,47 & 24,48 & 24,55 & 0,79 & 3,23 \\
\hline S2 & 24,48 & 24,13 & 23,18 & 24,08 & 23,97 & 0,55 & 2,31 \\
\hline S3 & 24,14 & 21,41 & 29,19 & 27,15 & 25,47 & 3,41 & 13,39 \\
\hline S4 & 25,65 & 25,15 & 19,91 & 23,38 & 23,52 & 2,60 & 11,04 \\
\hline S5 & 21,94 & 20,63 & 17,32 & 20,29 & 20,05 & 1,95 & 9,73 \\
\hline S6 & 19,71 & 20,8 & 43,08 & 29,21 & 28,20 & 10,79 & 38,26 \\
\hline Média & 23,54 & 22,85 & 26,03 & 24,77 & & & \\
\hline Desvio & 2,28 & 2,13 & 9,26 & 3,10 & & & \\
\hline CV (\%) & 9,69 & 9,30 & 35,58 & 12,50 & & & \\
\hline
\end{tabular}

$K_{1}^{\prime}$ valor estimado por regressão para o modelo $y=K \cdot V_{a p}{ }^{b} ; K^{\prime}{ }_{2}$ obtido pela média de três repetições da dimensão medida no campo para $V_{a p}$ de $1,0 \mathrm{~L} \mathrm{~h}^{-1}, K^{\prime}{ }_{3}$ e $K^{\prime}{ }_{4}$ são coeficientes angulares das retas $y=a+b x$ e $y=b x$, respectivamente, com $y$ sendo a dimensão do bulbo e $x$ o valor de $V_{a p}^{1 / 3}, V_{a p}^{2 / 5}$ e $V_{a p}{ }^{1 / 4}$ para $D_{\max }, Z_{\max }$ e $D_{s}$, respectivamente. S1 - Luvissolo Crômico, S2 - Argissolo Vermelho-Amarelo, S3 - Cambissolo Háplico, S4 - Neossolo Quartzarênico, S5 - Latossolo Vermelho e S6 - Neossolo Flúvico.

Avaliando os valores de $K_{1}^{\prime}, K_{2}^{\prime}{ }_{2} K^{\prime}{ }_{3}$ e $K^{\prime}{ }_{4}$ dentro de cada solo, observa-se que os menores valores de CV foram observados para $D_{\max }$ quando comparado com a variabilidade dos valores de $\mathrm{K}^{\prime}$ para $Z_{\max }$ e $D_{s}$. O 
menor valor de CV para $D_{\max }$ foi observado usando $K_{4}^{\prime}$ com CV de 2,56\%, enquanto para $Z_{\max }$ e $D_{s}$ os menores valores de CV foram para $K^{\prime}{ }_{1}$ e $K_{2}^{\prime}{ }_{2}$, com $15,66 \%$ e $6,30 \%$, respectivamente.

Avaliando os valores de $D_{\max }, Z_{\max }$ e $D_{s}$ estimados pelo modelo usando os diferentes valores de $K^{\prime}$ em função dos valores observados no campo, observa-se na Figura 1 que para $D_{\max }$, verificou-se boa relação entre o estimado e observado, com valores do coeficiente de determinação $\left(R^{2}\right)$ superior a 0,9600 para os solos avaliados, com exceção do Neossolo Flúvico (S6) cujo valor foi de 0,8956. Já para o $Z_{\max }$ (Figura 2), o $\mathrm{R}^{2}$ foi de 0,7519 para o $\mathrm{S} 6$ e superiores a 0,92 para os demais solos. Para o $D_{s}$ (Figura 3), estes valores foram superiores a 0,89 para todos os solos, com exceção do $S 6$ com $R^{2}$ de 0,6555 .
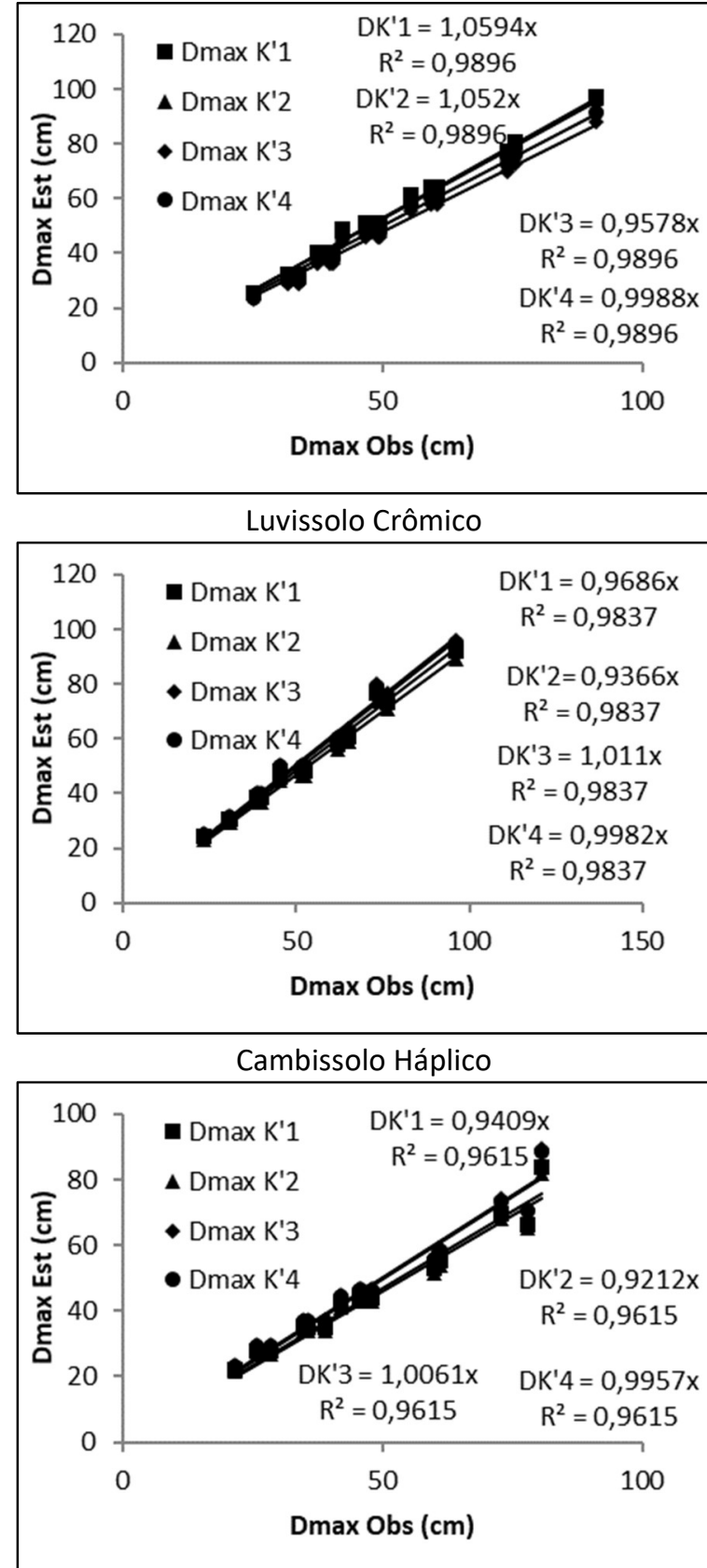

Latossolo Vermelho

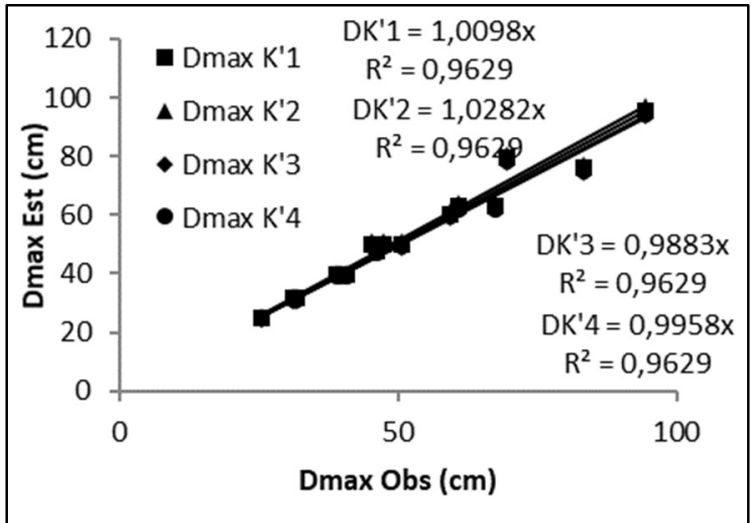

Argissolo Vermelho-Amarelo

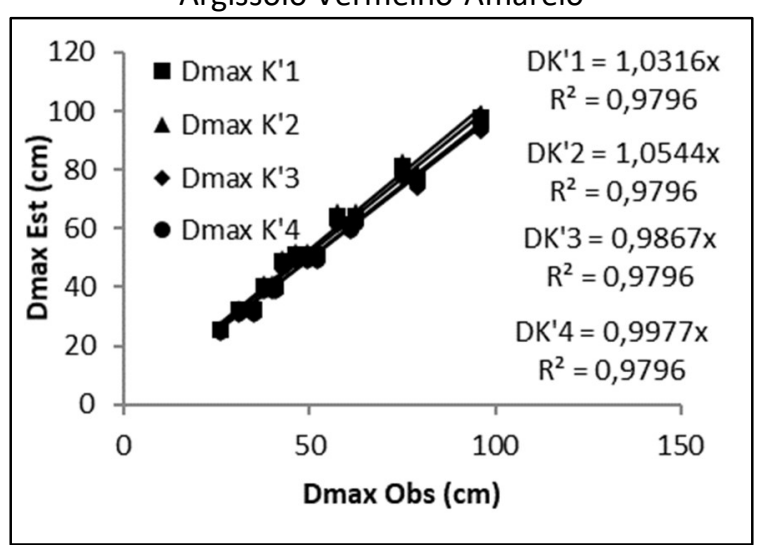

Neossolo Quartzarênico

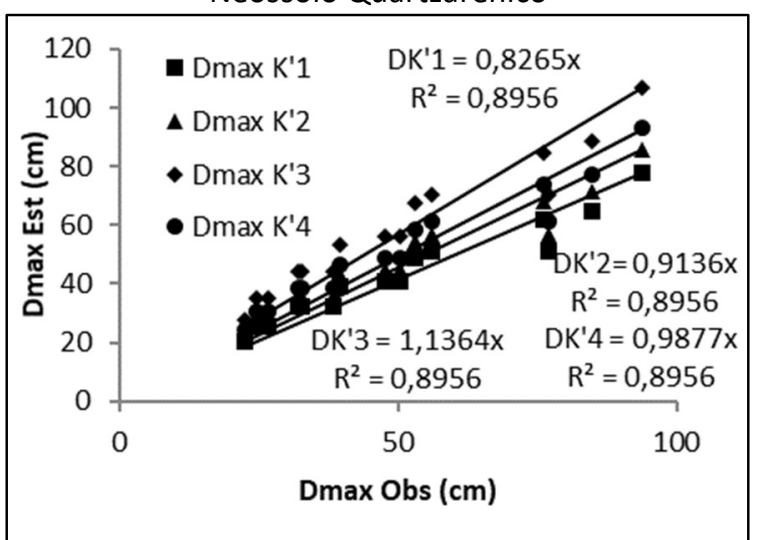

Neossolo Flúvico

Figura 1: Diâmetro máximo estimado pelo modelo $(\mathrm{cm})$ em função dos valores observados para os solos avaliados. 


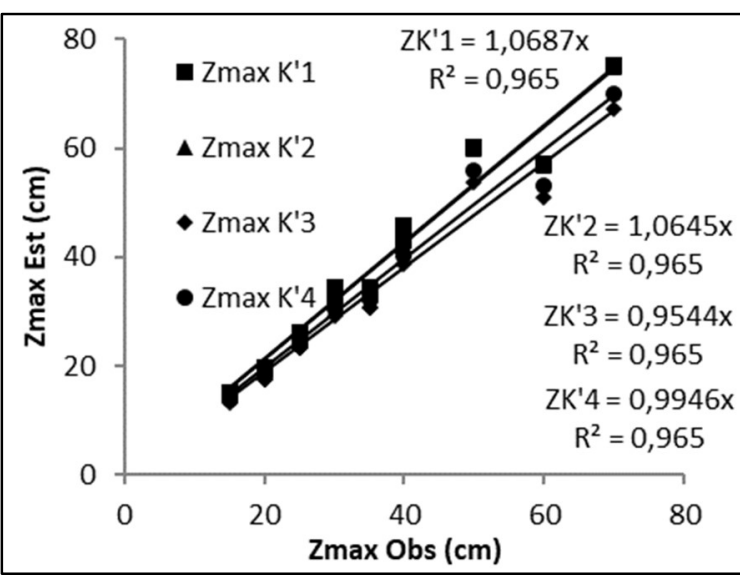

Luvissolo Crômico

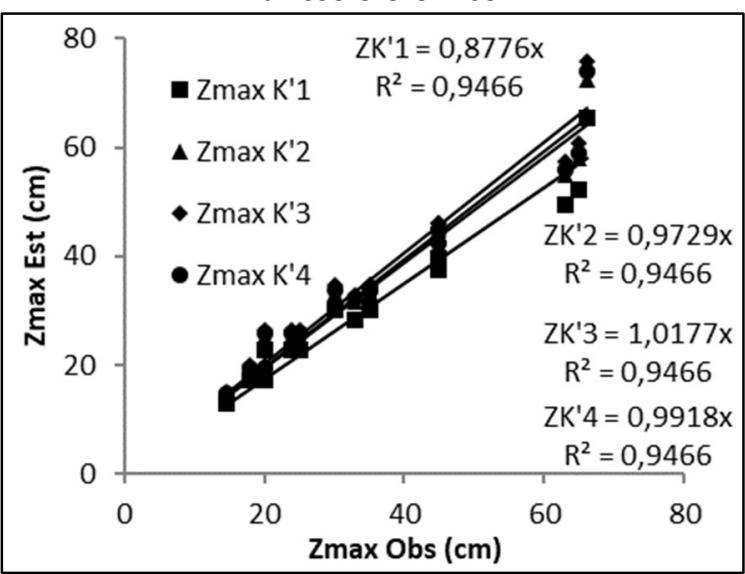

Cambissolo Háplico

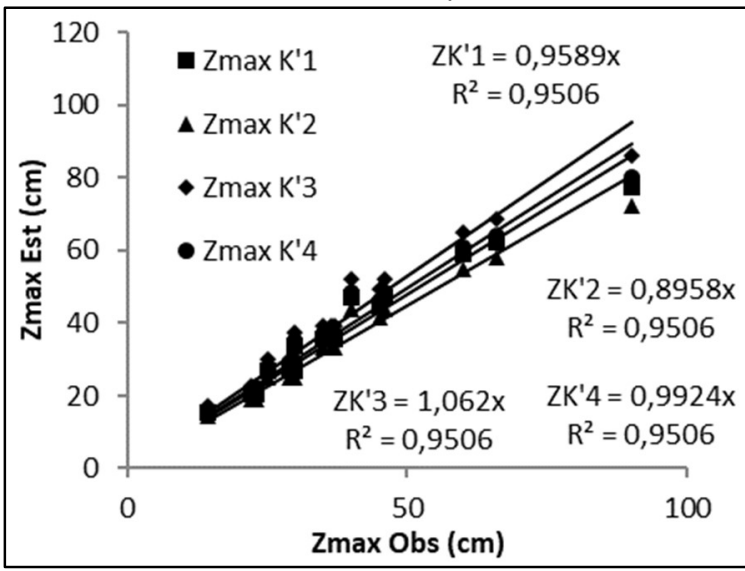

Latossolo Vermelho

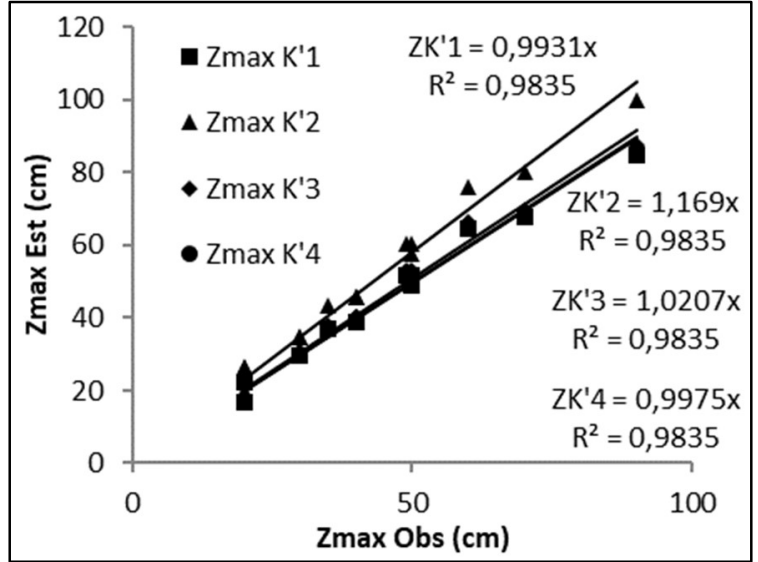

Argissolo Vermelho-Amarelo

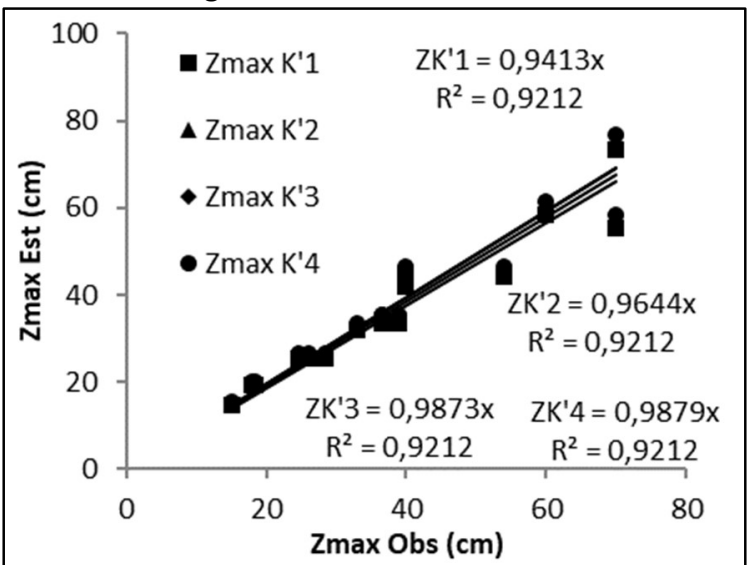

Neossolo Quartzarênico

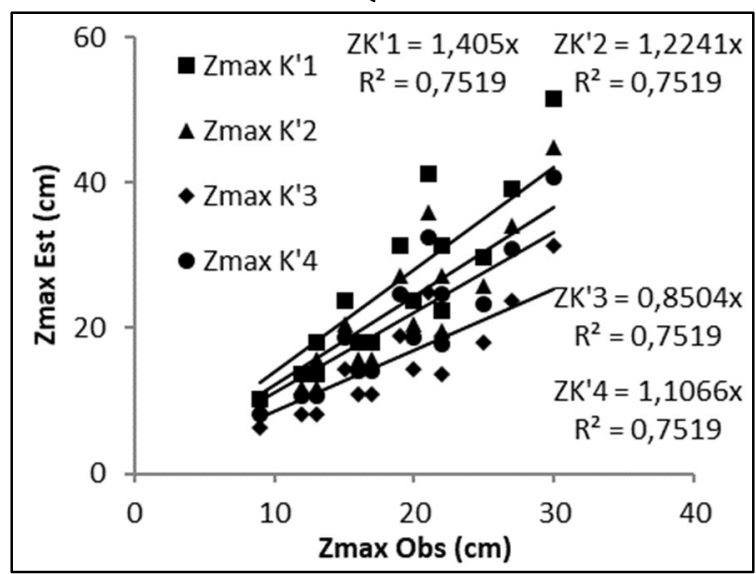

Neossolo Flúvico

Figura 2: Profundidade máxima estimada pelo modelo $(\mathrm{cm})$ em função dos valores observados para os solos avaliados.

Apesar do bom ajuste observado, outra maneira de avaliar a eficiência dos modelos matemáticos é a declividade da reta que estima os valores estimados em função dos observados, sendo o ideal que este valor (coeficiente angular) seja igual a 1,0, onde os valores estimados seriam iguais aos observados. Nesse sentido, observado os valores da declividade das retas nas Figuras 1, 2 e 3, observa-se para cada solo que este foi próximo a 1,0 com exceção do Neossolo Flúvico cujos coeficientes variaram de 0,8265 $\left(K_{1}^{\prime}\right)$ a 1,1364 $\left(K_{3}^{\prime}\right)$ para $D_{\max }$, de 0,8504 $\left(K^{\prime}{ }_{3}\right)$ a 1,4050 $\left(K_{1}^{\prime}\right)$ para $Z_{\max }$ e de 0,6604 $\left(K_{1}^{\prime}\right)$ a 0,8681 $\left(K_{4}^{\prime}\right)$ para $D_{s}$. Assim, mesmo os valores dos coeficientes angulares se aproximarem de 1,0 para os demais solos, observa-se que aos mais próximos valores da unidade são verificados quando se usa o valor de $K_{4}^{\prime}$, em que, até para $D_{s}$ no solo $\mathrm{S} 6$, apesar do valor de 0,8681, este foi o mais próximo de 1,0. 
Dentre os métodos avaliado aqui para estimar $K^{\prime}$, para a estimativa do $K^{\prime}{ }_{1}$, para uma boa estimativa de $K^{\prime}{ }_{1}$ é necessária a avaliação de pelo menos cinco volumes de água aplicado com pelo menos três repetição. Com base nos dados de Maia (2011), poderia avaliar os $V_{a p}$ de 1, 8, 14, 32 e 56 L levando até 7 h de aplicação para aplicar $56 \mathrm{~L}$ de água para uma vazão do emissor de $8 \mathrm{~L} \mathrm{~h}^{-1}$. Da mesma forma para a estimativa de $K^{\prime}{ }_{3} \mathrm{e}$ $K^{\prime}{ }_{4}$, levando em consideração que a relação usada para obter esses parâmetros é linear, está poderia também ser estimada a partir das vazões utilizada para estimar $K^{\prime}{ }_{1}$. Já para a estimativa de $K^{\prime}{ }_{2}$ esta seria a mais simples, sendo necessário apenas obter valores das medidas de $D_{\max }, Z_{\max }$ e $D_{s}$ em condições de campo obtidas pela média de pelo menos três repetições para infiltração do volume de água aplicado $\left(V_{a p}\right)$ de $1,0 \mathrm{~L}$.

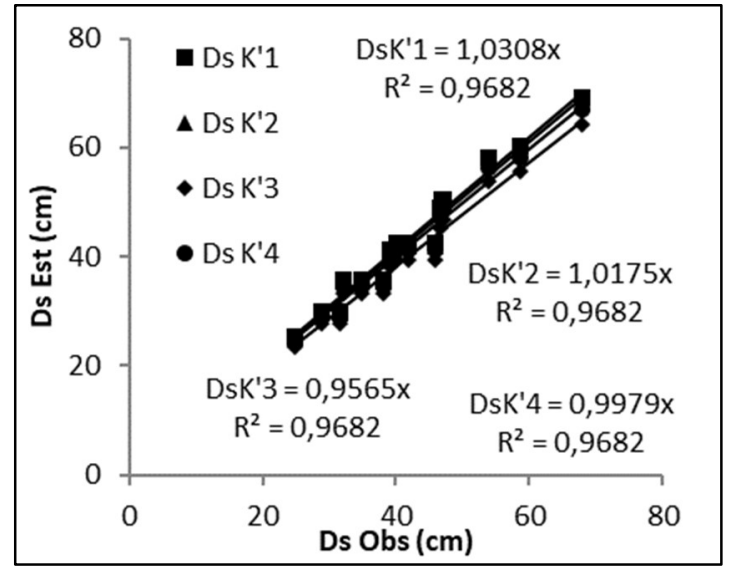

Luvissolo Crômico

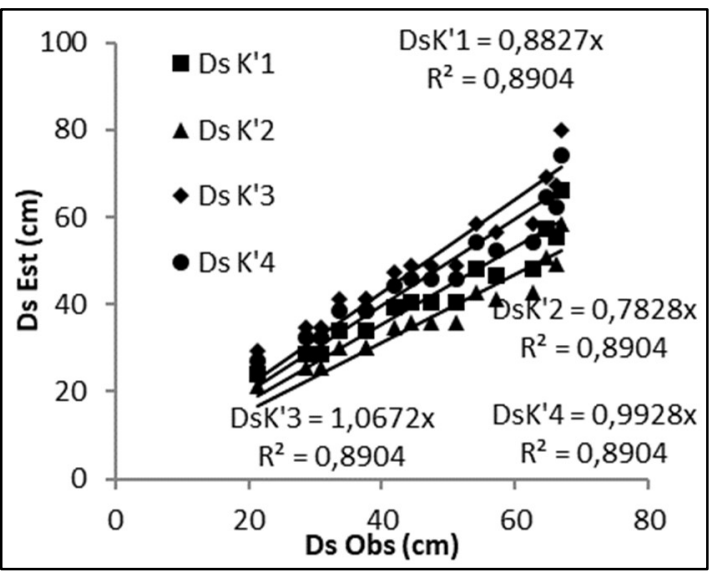

Cambissolo Háplico

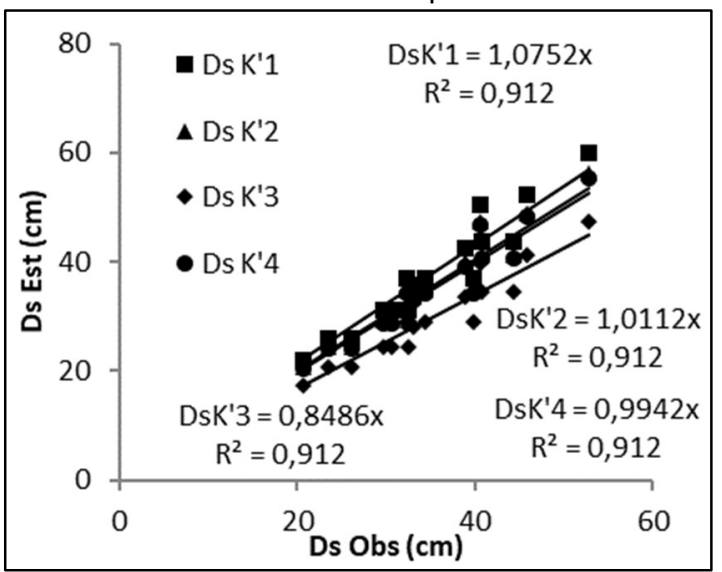

Latossolo Vermelho

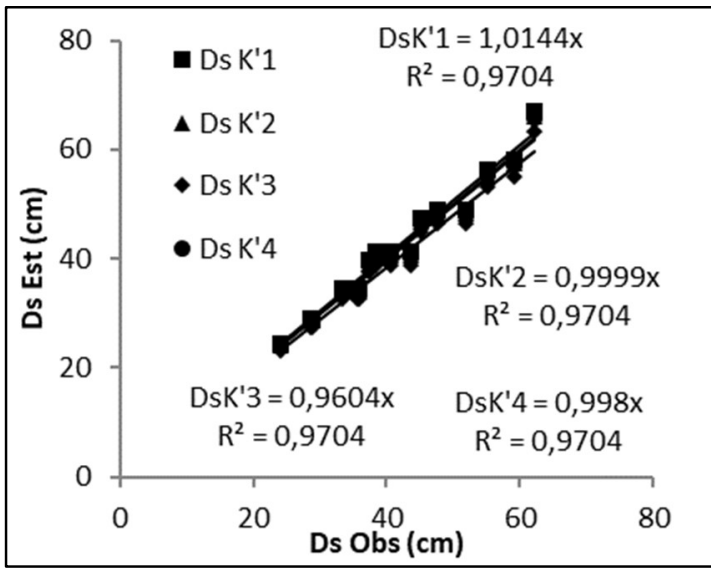

Argissolo Vermelho-Amarelo

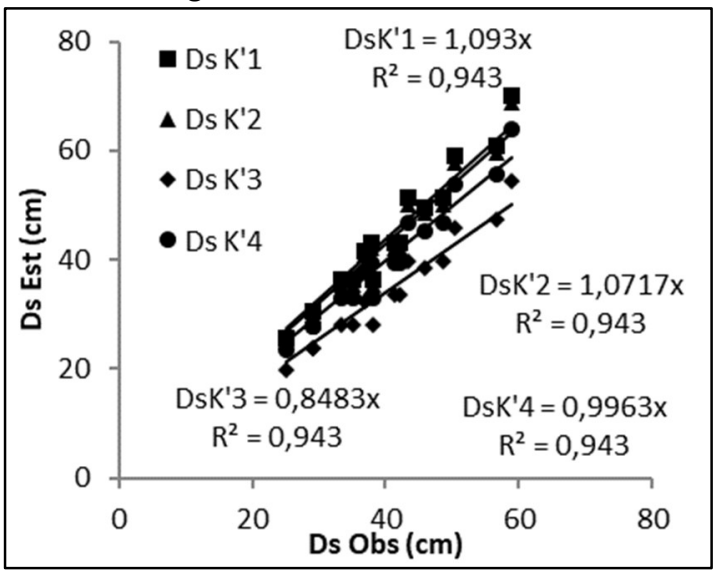

Neossolo Quartzarênico

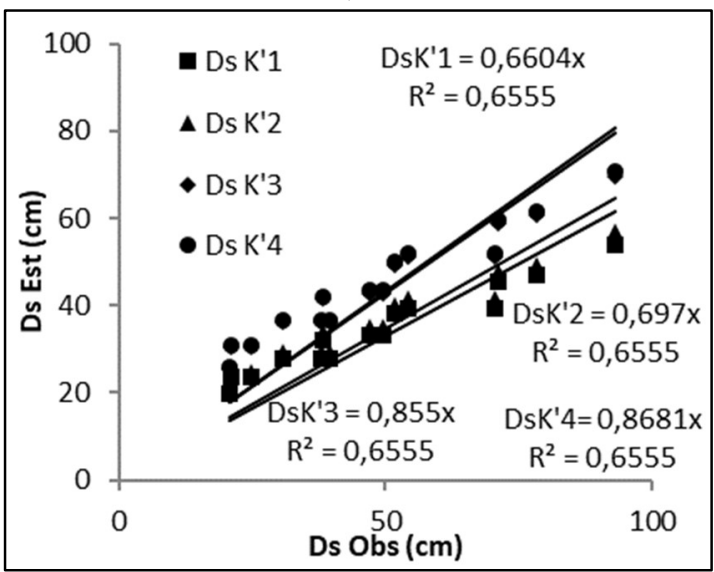

Neossolo Flúvico

Figura 3: Diâmetro superficial estimado pelo modelo $(\mathrm{cm})$ em função dos valores observados para os solos avaliados. 


\section{CONCLUSÕES}

O modelo potencial com estimativa de apenas um parâmetro pode ser utilizado para estimar os valores de $D_{\max }, Z_{\max }$ e $D_{s}$ em função do volume de água aplicado na irrigação por gotejamento superficial. Os quatro métodos para estimar o parâmetro $K^{\prime}$ podem ser utilizados, porém, o método para obter $K_{2}^{\prime}$ é o mais simples e com boa exatidão.

\section{REFERÊNCIAS}

CARMO FILHO, F.; ESPÍNOLA SOBRINHO J.; MAIA NETO J. M.. Dados climatológicos de Mossoró: um município semiárido nordestino. Mossoró: ESAM, 1991.

CHANDRAMOHAN, P.; BALAMURUGAN, P.; DINESH, S.. Effect offluidflow diversion and venturi size on drip irrigation fertilizer feeding system. Flow Measurement and Instrumentation, v.65, p.250-256, 2019. DOI: https://doi.org/10.1016/i.flowmeasinst.2019.01.015

COOK, F. J.; THORBURN, P. J.; FITCH, P.; BRISTOW, K. L.. Wet Up: a software tool to display approximate wetting patterns from drippers. Irrigation Science, v.22, p.129-134, 2003. DOI: http://doi.org/10.1007/s00271-003-0078-2

HAO, A.; MARUI, A.; HARAGUCHI, T.; NAKANO, Y.. Estimation of wet bulb formation in various soil during drip irrigation. Journal of the Faculty of Agriculture, v.52, n.1, p.187-193, 2007.

KANG, S.; HAO, X.; DU, T.; TONG, L.; SU, X.; LU, H.; LI, X.; HUO, Z.; LI; S.; DING, R.. Improving agricultural water productivity to ensure food security in China under changing environment: from research to practice. Agricultural Water Management, v.179, p.5-17, 2017. DOI:

https://doi.org/10.1016/j.agwat.2016.05.007

LI, J.; ZHANG, J.; RAO, M.. Wetting patterns and nitrogen distributions as affected by fertigation strategies from a surface point source. Agricultural Water Management, v.67, p.89-104, 2004. DOI:

https://doi.org/10.1016/j.agwat.2004.02.002

MA, X.; SANGUINET, K. A.; JACOBY, P. W.. Direct root-zone irrigation outperforms surface drip irrigation for grapeyield and crop water use efficiency while restricting root growth. Agricultural Water Management, v.231, p.105993, 2020. DOI: https://doi.org/10.1016/j.agwat.2019.105993

MAIA, C. E.. Modelos matemáticos para estimativa da geometria do volume de bulbo molhado por irrigação por gotejamento superficial no Agropolo Assu-Mossoró. Tese (Doutorado em Recursos Naturais) - Universidade Federal de Campina Grande, Campina Grande, 2005.

MAIA, C. E.; LEVIEN, S. L. A.. Estimativa de dimensões de bulbo molhado em irrigação por gotejamento superficial aplicando modelo de superfície de resposta. Ciência Rural, v.40, n.6, p.1302-1308, 2010. DOI: https://doi.org/10.1590/S0103-84782010005000099

MAIA, C. E.. Dimensões do bulbo molhado na irrigação por gotejamento superficial em função do volume de água aplicado. In: REUNIÃO SUL-AMERICANA PARA MANEJO E SUSTENTABILIDADE DA IRRIGAÇÃO EM REGIÕES ÁRIDAS E SEMIÁRIDAS, 2. Anais. Cruz das Almas: UFBA, 2011.

MAIA, C. E.; LEVIEN, S. L. A.; MEDEIROS, J. F.; DANTAS NETO, J.. Dimensões de bulbo molhado na irrigação por gotejamento superficial. Revista Ciência Agronômica, v.41, n.1, p.149-158, 2010.

SANTOS, H. G.; JACOMINI, P. L. T.; ANJOS, L. H. C.; OLIVEIRA, V. A.; LUMBRERAS, J. F.; COELHO, M. R.; ALMEIRA, J. A.; CUNHA, T. J. F.; OLIVEIRA, J. B.. Sistema brasileiro de classificação de solos. 3 ed. Brasília: Embrapa, 2013.

SCHWANKL, L. J.; HANSON, B. R.. Surface Drip Irrigation. Developments in Agricultural Engineering, v.13, n.7, p.431472, 2007. DOI: https://doi.org/10.1016/S01674137(07)80015-1

SCHWARTZMAN, M.; ZUR, B.. Emitter spacing and geometry of wetted soil volume. Journal of Irrigation and Drainage Engineering, v.112, n.3, p.242-253, 1986.

THABET, M.; ZAYANI, K.. Wetting patterns under trickle source in a loamy sand soil of south Tunisia. AmericanEurasian Journal of Agricultural \& Environmental Sciences, v.3, n.1, p.38-42, 2008.

THORBURN, P. J.; COOK, F. J.; BRISTOW, K. L.. Soil-dependent wetting from trickle emitters: implications for system design and management. Irrigation Science, v.22, p.121-127, 2003. DOI: https://doi.org/10.1007/s00271-003-0077-3

YANG, D.; LI, S.; KANG, S.; DU, T.; GUO, P.; MAO, X.; TONG, L.; HAO, X.; DING, R.; NIU, J.. Effect of drip irrigation on wheat evapotranspiration, soil evaporation and transpiration in Northwest China. Agricultural Water Management, v.232, p.106001, 2020. DOI: https://doi.org/10.1016/j.agwat.2020.106001

A CBPC - Companhia Brasileira de Produção Científica (CNPJ: 11.221.422/0001-03) detém os direitos materiais desta publicação. Os direitos referem-se à publicação do trabalho em qualquer parte do mundo, incluindo os direitos às renovações, expansões e disseminações da contribuição, bem como outros direitos subsidiários. Todos os trabalhos publicados eletronicamente poderão posteriormente ser publicados em coletâneas impressas sob coordenação da Sustenere Publishing, da Companhia Brasileira de Produção Científica e seus parceiros autorizados. Os (as) autores (as)

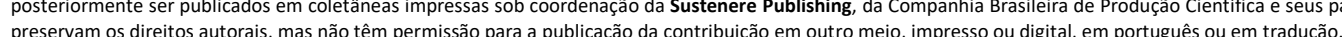

